\title{
Fast destruction of singlet order in NMR experiments
}

Bogdan A. Rodin, ${ }^{1,2}$ Kirill F. Sheberstov, ${ }^{1,3}$ Alexey S. Kiryutin,, ${ }^{1,2}$ Lynda J. Brown, ${ }^{4}$ Richard C. D. Brown, ${ }^{4}$ Mohamed Sabba, ${ }^{4}$ Malcolm H. Levitt, ${ }^{4}$ Alexandra V.

Yurkovskaya, ${ }^{1,2}$ and Konstantin L. Ivanov ${ }^{1,2, a)}$

1) International Tomography Center, Siberian Branch of the Russian Academy of Science, Novosibirsk 630090, Russia

${ }^{2)}$ Novosibirsk State University, Novosibirsk 630090, Russia

3) Johannes Gutenberg-Universitat, Helmholtz Institute Mainz, 55099, Germany

4) Department of Chemistry, Southampton University, Southampton SO17 1BJ, $U K$

(Dated: 26 November 2019)

Some nuclear spin systems support long-lived states, which display greatly extended relaxation times relative to the relaxation time of nuclear spin magnetization. In spin-1/2 pairs, the such a long-lived state is given by singlet order, representing the difference of the population of the nuclear singlet state and the mean population of the three triplets. In many cases, the experiments with long-lived singlet order are very time-consuming because of the need to wait for singlet order decay before the experiment can be repeated; otherwise spin order remaining from a previous measurement may lead to experimental artefacts. Here we propose techniques for fast and efficient singlet order destruction. These methods exploit coherent singlet-triplet conversion; in some cases, multiple conversion steps are introduced. We demonstrate that singlet order destruction enables a dramatic reduction of the waiting time between consecutive experiments and suggest to use this approach in singlet-state NMR experiments with nearly equivalent spins.

a)ivanov@tomo.nsc.ru 


\section{INTRODUCTION}

Experiments with nuclear singlet spin order ${ }^{1-3}$ are attracting increased attention in $\mathrm{Nu}-$ clear Magnetic Resonance (NMR). In systems of spin-1/2 pairs, singlet order, representing the population imbalance between the singlet and triplet states, is often long-lived, in the sense that its relaxation time $T_{S}$ is much longer than the relaxation time $T_{1}$ of the longitudinal nuclear spin magnetization. The increased $T_{S}$ lifetime is due to the fact that nuclear singlet order is protected against some of the relaxation mechanisms. Specifically, singlet order is immune to relaxation driven by the intra-pair dipole-dipole coupling, which often gives the dominant mechanism for $T_{1}$ relaxation. Very large ratios $T_{S} / T_{1}$ have been demonstrated in various cases $^{4-6}$. The extended lifetime of nuclear singlet spin order paves the way to a number of new NMR applications, for example, to studies of slow processes ${ }^{7-14}$, characterization of protein-ligand binding ${ }^{12}$ and storage or transport of enhanced nuclear spin polarization ${ }^{6,14-23}$. The mechanisms of singlet order decay have been studied in some detail $^{4,24}$ revealing the role of various fluctuating interactions, e.g., chemical shift anisotropy, spin-rotational interaction, etc.

A special case of systems possessing a long-lived singlet state is given by molecules with pairs of nearly-equivalent spins, in which the non-equivalency is achieved by a small chemical shift difference ${ }^{4}$. In this case the J-coupling is much greater than the difference in the NMR frequencies of the two nuclei. Consequently, the singlet state is almost an eigenstate of the system even at a high external magnetic field. A similar situation is met when the two spins are chemically equivalent but magnetically non-equivalent ${ }^{25-27}$, due to a small difference in J-couplings to other spins in the molecule. Remarkably, in systems of nearly-equivalent spins the long-lived state can be sustained even at high fields in the absence of spin-locking ${ }^{4}$.

Since the singlet state is nearly an eigenstate of the system, experiments with pairs of nearly equivalent spins require special care. For instance, generation and readout of the singlet state should be done using special pulse sequences. The known NMR methods for magnetization-to-singlet (M2S) conversion (utilized to generate the singlet order) and reverse singlet-to-magnetization (S2M) conversion (used for singlet-state readout) in such spin pairs include a CPMG-based NMR pulse sequence with pulses synchronized with the J-driven spin evolution ${ }^{28}$, SLIC (Spin-Locking Induced Crossing) method ${ }^{29}$ and APSOC (AdiabaticPassage Spin Order Conversion) method ${ }^{30,31}$. However, since singlet order relaxes very 
slowly, the repetition rate of NMR experiments becomes low: in general, the delay between subsequent NMR experiments should be long enough that not only spin magnetization but also the singlet order relax to their equilibrium values. In practice, such delays should be of the order of $3 T_{S}$ to $5 T_{S}$, instead of the much shorter delay of $3 T_{1}$ to $5 T_{1}$, sufficient for the spin magnetization to relax. For very long $T_{S}$ relaxation times, which are of interest for many applications, the relaxation delay becomes so long that repetitive experiments might become very time consuming.

In this work, we propose a family of methods aimed at fast Singlet Order Destruction (SOD) in pairs of nearly equivalent spins. The SOD block can be combined with singletstate NMR experiments in a straightforward way to speed them up. We demonstrate fast destruction of singlet order under the action of the SOD techniques and also a significant reduction of the measurement time using two examples. The first one is dealing with excitation of the "forbidden" transitions ${ }^{32}$ in a strongly-coupled spin pair: in these experiments we analyze the nutation curves measured after excitation by a weak selective NMR pulse. We clearly demonstrate that the proposed SOD schemes provide a strong reduction of the repetition time also avoiding the detrimental influence from the previous experiment. The second example is given by measurements of the typical singlet order decay kinetics where the SOD block also enables a similar reduction of the total time of the experiment.

\section{THEORY}

In this work, we aim to destroy singlet spin order, which is defined as the singlet-triplet population imbalance:

$$
S O=P_{S}-\left\langle P_{T}\right\rangle, \text { where }\left\langle P_{T}\right\rangle=\frac{1}{3}\left\{P_{T_{+}}+P_{T_{0}}+P_{T_{-}}\right\}
$$

Here $P_{i}$ is the population of the corresponding state; $\left\langle P_{T}\right\rangle$ is thus the average population of the three triplet states. To perform fast singlet order destruction we propose a SOD technique presented in Figure 1. The idea of SOD is repetition of the following two steps. The first step is equalizing the populations of the triplet states. According to eq. (1) and the fact that $\sum_{i} P_{i}=1$, the populations after equalization are defined as

$$
P_{S}=\frac{3 \cdot S O+1}{4} ; \quad\left\langle P_{T}\right\rangle=\frac{1-S O}{4}
$$


The second step is exchanging the populations of the singlet state and one of the triplet states. In this particular case, it does not matter, which of the three triplet states is chosen, as they all have the same population. For the sake of generality, we assume that exchange of populations is not necessarily complete. Hence, we introduce the population exchange efficiency, by presenting the population exchange as a rotation on the Bloch sphere by the angle $\theta$; here $\theta=\pi$ corresponds to complete exchange of the populations. After rotation, the singlet state population $P_{S}^{\prime}$ becomes equal to

$$
P_{S}^{\prime}=P_{S} \cos ^{2} \frac{\theta}{2}+\left\langle P_{T}\right\rangle \sin ^{2} \frac{\theta}{2}
$$

By using eq. (1) we rewrite this expression as

$$
P_{S}^{\prime}=\frac{1}{4}+\frac{S O}{4}\left(4 \cos ^{2} \frac{\theta}{2}-1\right)
$$

and the singlet order becomes $S O^{\prime}=\left(4 P_{S}^{\prime}-1\right) / 3$. By using eq. (4) we obtain

$$
S O^{\prime}=\frac{S O}{3}\left(4 \cos ^{2} \frac{\theta}{2}-1\right)
$$

Hence, we can see that when $\cos \frac{\theta}{2}=\frac{1}{2}$, i.e., $\theta=\frac{2 \pi}{3}$, singlet order becomes zero independent of its initial value. It is worth mentioning that some techniques allow one to set the $\theta$ angle equal to a value of choice, whereas, e.g., in APSOC, it is hard to set $\theta$ equal to a precise value (when APSOC pulses are optimized $\theta$ is close to $\pi$ ). Nonetheless, the SOD scheme is still applicable: when $\theta \neq \frac{2 \pi}{3}$ SOD can be implemented by repeating the cycle (conversion)(triplet equalization) multiple times. In such a scheme, singlet order decays exponentially with the number of cycles, $n$. For instance, when $\theta=\pi$ (exact exchange of populations) the $S O$ dependence on $n$ is as follows:

$$
S O(n)=\frac{1}{(-3)^{n}} S O_{i n i}
$$

where $S O_{i n i}$ is the initial spin order. Thus, when $\theta=\pi$ we obtain $S O(n=1)=-S O_{i n i} / 3$, $S O(n=2)=S O_{i n i} / 9$, etc. The SOD procedure should be repeated until singlet order is completely erased.

The SOD block can be integrated in protocols of NMR experiments with singlet spin order. In this work, we analyze how singlet order decays upon application of the SOD block and also demonstrate how the proposed method can be used to speed up experiments involving long-lived singlet order. 

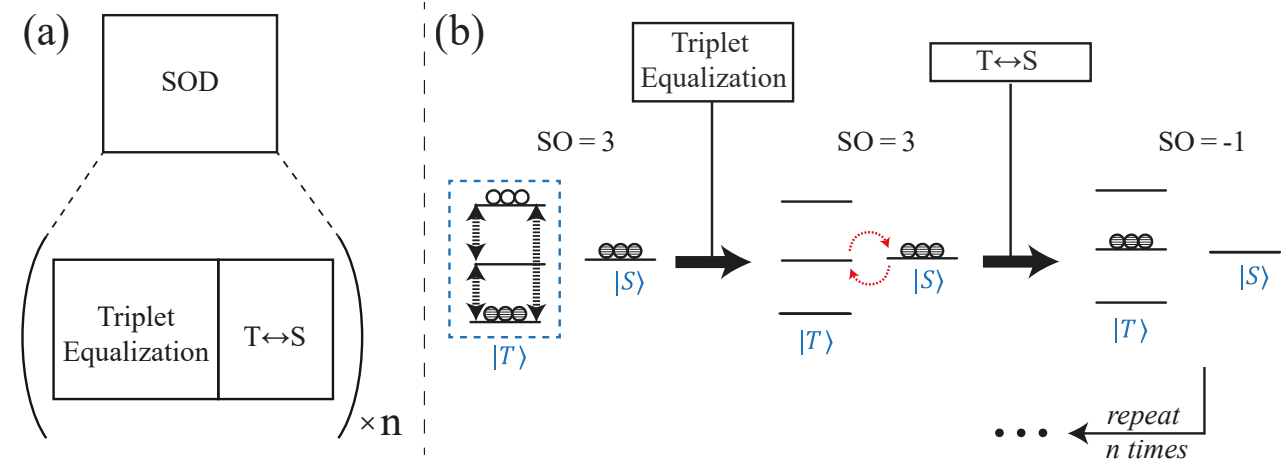

FIG. 1. Singlet Order Destruction (SOD) scheme proposed in this work. The SOD block (subplot a) includes equalization of the triplet state populations, followed by an $T \leftrightarrow S$ conversion element. After triplet equalization and conversion (subplot b) singlet order becomes smaller. When the rotation angle is $\theta=\frac{2 \pi}{3}$ complete singlet order destruction will occur in single step, i.e., $n=1$. Otherwise, the sequence should be repeated until complete singlet order destruction.

Another approach to singlet order destruction is given by dynamic coupling of the longlived singlet state with the fast-relaxing triplet manifold. A possible method is to apply a pseudo-cw SLIC pulse ${ }^{29}$, which is a resonant radiofrequency (RF) pulse with the amplitude matched to $J$. As a result, the singlet order can be destroyed on the timescale of $T_{1}$. This method is not nearly as fast and efficient as the SOD method; for this reason, it is discussed only in Supplementary Material.

\section{METHODS}

\section{A. Sample preparation}

All experiments were performed using a specially designed naphthalene derivative ${ }^{4}$ with two ${ }^{13} \mathrm{C}$ labels (hereafter, ${ }^{13} \mathrm{C}_{2}-\mathrm{I}$ ), which constitute a pair of nearly equivalent spins possessing a long-lived singlet state. To prepare the sample, we place an acetone solution of ${ }^{13} \mathrm{C}_{2}$-I in a sealed microcell insert, which was positioned in a standard $5 \mathrm{~mm}$ NMR tube and fixed approximately $1 \mathrm{~cm}$ above the bottom. The NMR tube with the insert inside was filled with protonated acetone and sealed. As a result, the entire sample is located inside the NMR coil and high homogeneity of both static $B_{0}$ field and RF-field is achieved ${ }^{32,33}$.

The structure and NMR spectrum of ${ }^{13} \mathrm{C}_{2}$-I in deuterated acetone are shown in Figure 2. 
From the spectrum, one can readily see that the spins are strongly coupled, i.e., the coupling strength, $J$, is much greater than the difference, $\delta \nu$, in their Zeeman interactions with the $B_{0}$ field. As a result, in the spectrum there are two strong lines in the center of the spectrum (with the splitting between them equal to approximately $\delta \nu^{2} / 2 J$ ) and two weak satellites, which are spaced by $\approx J$ from the center of the spectrum ${ }^{4}$. In the present case, at the magnetic field $B_{0}=16.4 \mathrm{~T}$ the satellites are approximately 120 times weaker than the central lines. The satellites correspond to NMR transitions between the outer triplet states, $\left|T_{ \pm}\right\rangle$, and the state, which is only slightly different from $|S\rangle$ : the intensity of these lines is thus strongly reduced as singlet-triplet transitions are forbidden transitions in NMR. Due to the fact that the spin system is strongly coupled, singlet order can be sustained for a time period of $T_{S} \gg T_{1}$ even at high field in the absence of spin-locking; in the present case, $T_{S} \approx 30 T_{1}$.

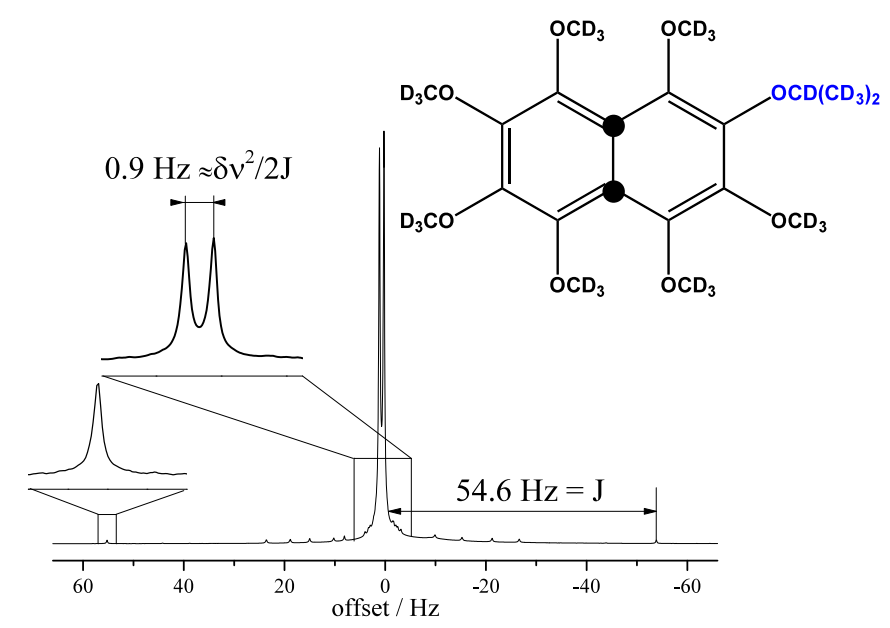

FIG. 2. Doubly ${ }^{13} \mathrm{C}$-labelled naphthalene derivative, ${ }^{13} \mathrm{C}_{2}$-I, with the ${ }^{13} \mathrm{C}$ labels highlighted and its ${ }^{13} \mathrm{C}$ NMR spectrum at $B_{0}=16.4 \mathrm{~T}$. In a $20 \mathrm{mM}$ solution in acetone- $\mathrm{d}_{6}{ }^{13} \mathrm{C}_{2}$-I has $T_{1}=7 \mathrm{~s}$ and $T_{S}=217 \mathrm{~s}$ at $B_{0}=16.4 \mathrm{~T}$ in the absence of spin-locking. The strong inner peaks of the NMR quartet are split by $0.9 \mathrm{~Hz}$ (see inset). One of the small outer peaks of the AB spectrum is enlarged. 


\section{B. NMR pulse sequences}

The blocks of the general SOD scheme illustrated by Figure 1 can be implemented in different ways. In this work, we used the following methods for triplet equalization and $T \leftrightarrow S$ conversion.

Triplet equalization. To equalize the populations of the triplet states, one can use a 90-degree pulse, which, however, generates unwanted spin coherences. In the simplest way, these coherences can be destroyed by applying a magnetic field gradient. A more complicated but also more efficient method makes use of the so-called $T_{00}$-filter, ${ }^{34,35}$ which wipes out any spin order but singlet order. The $T_{00}$-block comprises RF-pulses with properly set phases and magnetization flip angles and magnetic field gradients, see Figure 3(c). A comparison of the performance of the different triplet equalization elements is presented in Supplementary Material.

Triplet-singlet conversion. Spin order conversion can also be performed in various ways: here we consider in detail two conversion methods, APSOC and a J-synchronized CPMG-based sequence.

The APSOC method ${ }^{30,31,36}$, see Figure 3a, swaps the populations of the singlet state and a triplet state of choice by adiabatic correlation of states in the RF-rotating frame upon a slow increase of the RF-field strength, $\nu_{1}$. Indeed, is one of the eigenstates at $\nu_{1}=0$ is approximately equal to the singlet state, and it is correlated with the $\left|T_{+}\right\rangle$or $\left|T_{-}\right\rangle$state, which are the eigenstate at a high $\nu_{1}$. The conversion pathway, $|S\rangle \rightarrow\left|T_{+}\right\rangle$or $|S\rangle \rightarrow\left|T_{-}\right\rangle$, is chosen by setting the RF-frequency. To achieve a high degree of adiabaticity with a moderate pulse duration, we use optimized shapes ${ }^{33,37}$ of the APSOC pulses, introducing "constant adiabaticity" pulses. Finally, it is important to note that the APSOC pulse with an increasing $\nu_{1}(t)$ correlates the singlet state with the $\left|T_{ \pm}\right\rangle$states, as introduced in the "tilted" frame with the quantization axis parallel to the RF-field in the rotating frame.

In the J-CPMG method, see Figure 3b, we use a train of pulses, with the delays adjusted to the value of the $J$-coupling, $\tau_{J}=1 / 4 J$; here each pulse is a composite $180^{\circ}$ pulse. This pulse sequence performs a rotation in the space spanned by the $|S\rangle$ state and the central triplet $\left|T_{0}\right\rangle$ state. By changing the number of pulses, $n_{J}$, in the pulse train, it is possible to vary the rotation angle $\theta$. Details of this method are given elsewhere ${ }^{28}$. 


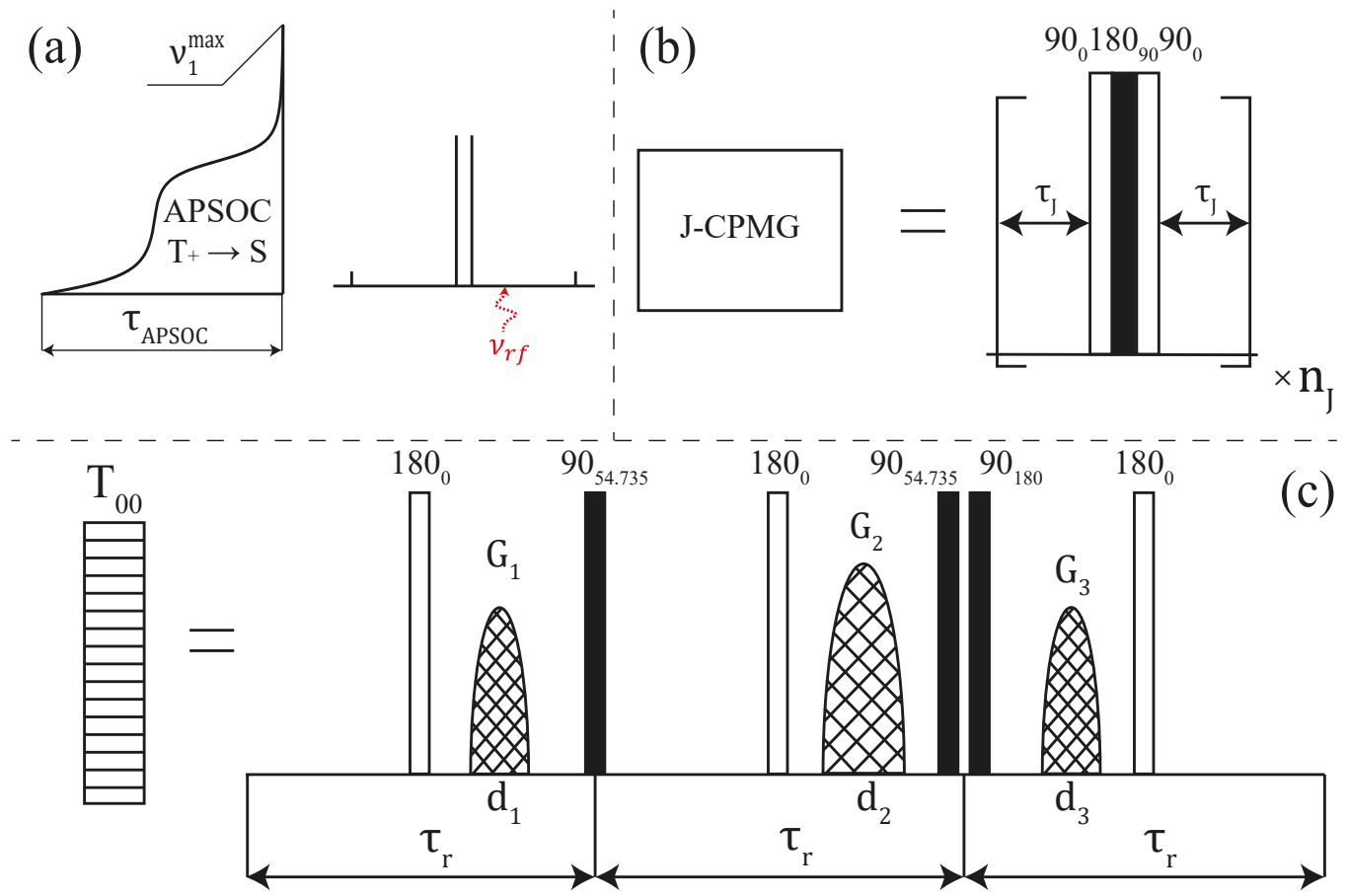

FIG. 3. Building blocks of the SOD pulse sequence. Subplots (a) and (b) schematically show the APSOC and J-CPMG sequences, respectively. The APSOC pulse (a) is a shaped pulse with adiabatically increased RF-field amplitude with a duration $\tau_{A P S O C}$, maximal amplitude $\nu_{1}^{\max }$ and frequency $\nu_{r f}$, which is slightly shifted from the center of the spectrum (as shown schematically by a wavy arrow). Parameters of the APSOC pulses used here: $\nu_{1}^{\max }=100 \mathrm{~Hz}$ (maximal strength of the RF-field), $\tau_{A P S O C}=0.34 \mathrm{~s}$ (pulse duration), $\Delta=10 \mathrm{~Hz}$ (off-set of the RF-field from the center of the spectrum). The J-CPMG block (b) comprises a train of composite $90_{0} 180_{90} 90_{0}$ pulses with a delay, $\tau_{J}$, matched to the J-coupling value; the cycle is applied $n_{J}$ times to achieve the desired degree of spin order conversion. Parameters of J-CPMG used here: $\tau_{J}=4.63 \mathrm{~ms}$ and $n_{J}=6$ (to achieve $\theta=\frac{2 \pi}{3}$ ) and $n_{J}=8$ (to achieve $\theta=\pi$. Subplot (c) shows the $T_{00}$ filter, which comprises RF-pulses and pulsed field gradients. The white and black boxes represent 90 and 180-degree pulses, respectively. The gradient durations are $\left\{d_{1}, d_{2}, d_{3}\right\}=\{4.4,2.4,2.0\} \mathrm{ms}$,

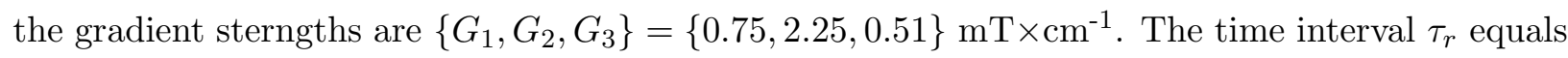
$1.8 \mathrm{~ms}$. The gradient duration was randomly varied with the amplitude $\pm 15 \%$ to avoid gradient correlation through $T_{00}$ repetition 


\section{RESULTS AND DISCUSSION}

\section{A. Singlet order destruction}

First of all, we demonstrate that the proposed SOD scheme enables singlet order destruction on a time scale much shorter than $T_{S}$ and even shorter than $T_{1}$. The timing scheme of the experiment is shown in Figure 4. First, the longitudinal magnetization is converted into singlet order by an APSOC pulse with an adiabatically ramped-up RF-field strength (M2S step). To estimate singlet spin order generated in this manner, we apply the $T_{00}$ filter ${ }^{34}$, followed by another APSOC pulse with an adiabatically decreased RF-field strength (S2M step). A $90^{\circ}$ pulse is applied to induce a free induction decay; the signal amplitude is determined from teh NMR spectrum obtained by Fourier transformation of the free induction decay. As shown before, ${ }^{33}$ such a scheme allows one measuring the time trace of singlet order relaxation. Note that in this experiment the APSOC pulses ${ }^{30,31,36}$ perform spin order conversion in the following way. The APSOC pulse with an adiabatically increased RF-field converts the population of the $\left|T_{+}\right\rangle$state (as defined in the frame with $z$ being the quantization axis) to that of the $|S\rangle$ state. The pulse with a decreased $\nu_{1}(t)$ amplitude works in the opposite way, performing the $S \rightarrow T_{+}$conversion and generating $z$-magnetization from singlet order.

Singlet lifetime measurements were performed by varying the relaxation delay between the APSOC pulses. In the present case we measure the decay time traces in the presence of a SOD block; hence, to increment the relaxation delay we vary the number of cycles, $n$, in this block. The resulting NMR signal is measured as a function of the number of cycles.

The singlet order decay curves are shown in Figure 5. One can see that all proposed schemes provide complete desctruction of singlet order on the timescale much shorter than $T_{S}$. In the case of exact population exchange, Figures $5 \mathrm{a}$ and $5 \mathrm{~b}$, the APSOC method and J-CPMG provide the same efficiency and the $n$-dependence is the same for both methods. Nevertheless, J-CPMG enables faster singlet order destruction because a single conversion step has a shorter duration. The $n$-dependence of singlet order contains oscillations coming from the fact that after each population swapping the $S O$ value changes the sign according to eq. (6). In each cycle, the $S O$ magnitude is reduced by approximately a factor of 3 .

The result for the SOD sequence using J-CPMG with the optimized rotation angle, 


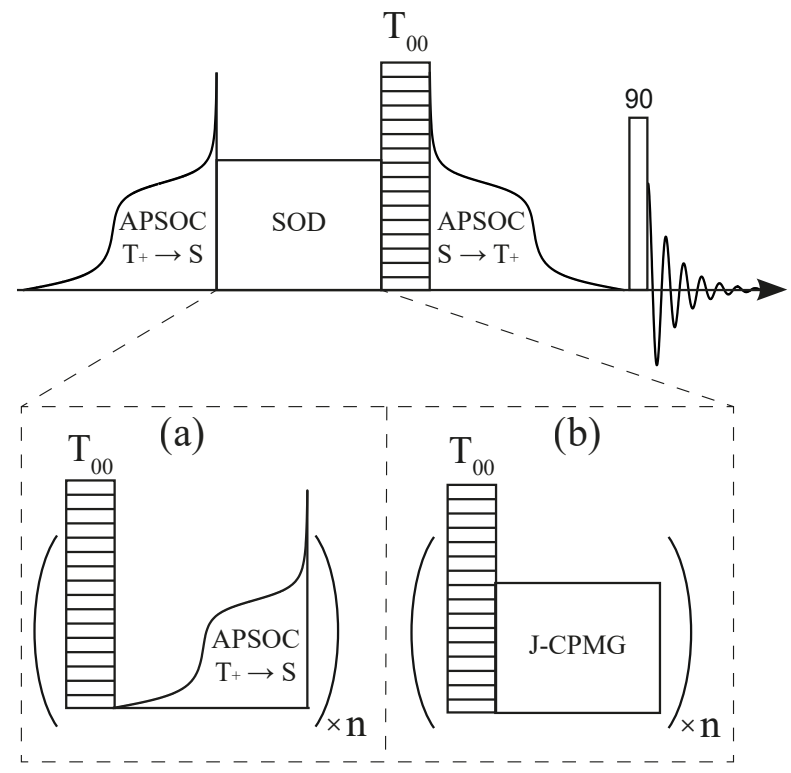

FIG. 4. Experimental protocol for measuring singlet order decay curve under the action of different SOD schemes. The number of the SOD cycles, $n$, is varied to increment the relaxation period. In case (a) the SOD block comprises the $T_{00}$ filter followed by an APSOC pulse, optimized for $T_{+} \rightarrow S$ conversion. In case (b) the $T_{00}$ filter is followed by a J-CMPG block optimized such that $\theta=\pi$ or $\theta=\frac{2 \pi}{3}$.

$\theta=\frac{2 \pi}{3}$, is shown on Figure 5c. Here we acheive an even faster destruction of singlet order, which almost completely disappears within a single cycle. Nevertheless, in the $n$-dependence one can see small fluctuations, presumably coming from partial refocusing of the spin order by magnetic field gradients, which are used in the $T_{00}$ filter. Despite this, the performance of this method is very good.

We compare the obtained relaxation curves with monoexponential curves, which decay with the characteristic relaxation times of the system under study, i.e., with $T_{1}$ and $T_{S}$ (see Figure 6, these two curves are not originating from an experiment but only schematically represent the decay of magnetization and singlet order). As one can see, the proposed SOD schemes wipe out the singlet order much faster than both $T_{1}$ and $T_{S}$.

Hence, our measurements clearly show that the proposed SOD method can swiftly erase the singlet order. Henceforth, we will use the SOD variation with the $T_{00}$ filter followed by an APSOC pulse. 


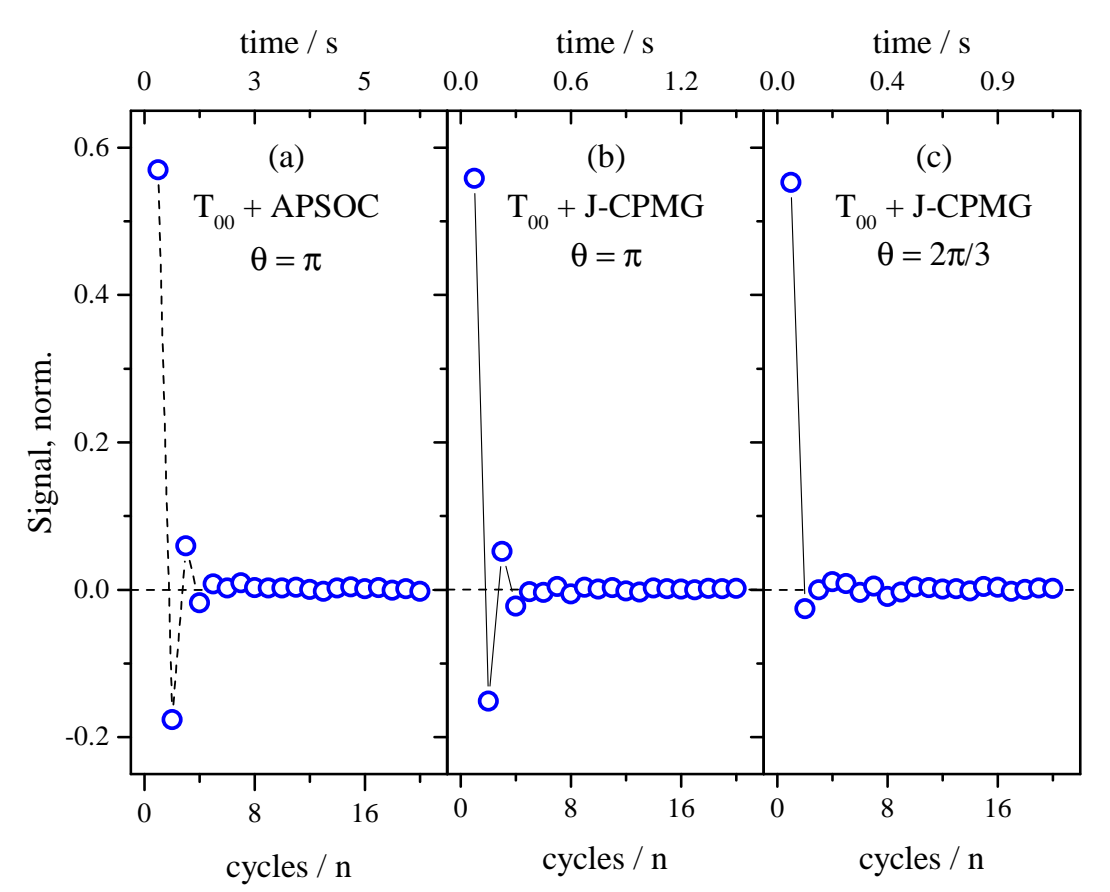

FIG. 5. Singlet order as a function of the number of SOD cycles, $n$, obtained using the protocol shown in Figure 4. In case (a) the SOD element consists of the $T_{00}$ filter followed by an APSOC-up pulse. In cases (b) and (c) the SOD element comprises the $T_{00}$ filter followed by a $J$-synchronized echo trains, which exchanges the populations of the $T_{0}$ and $S$ states. The number of echo cycles is 8 (b) and 6 (c). In all cases, the signal intensity is normalized by that of the thermal NMR signal.

\section{B. Measurement of the nutation curves for forbidden transitions}

In this subsection, we demonstrate how the SOD method can be applied to nutation experiments on forbidden transitions; such experiments are of interest for generating singlettriplet coherences. In this case, we excite the corresponding coherences ${ }^{32}$ by using a selective RF-pulse of a variable duration. The forbidden transitions are the transitions between the $\left|T_{ \pm}\right\rangle$states and the state, which almost coincides with $|S\rangle$. If the pulse is selective, which is guaranteed by using a low RF-field strength and a Gaussian pulse envelope, only the transition of interest is excited and the difference in the populations, $\left(P_{T_{+}}-P_{S}\right)$, is converted into the coherence, which is detected by measuring the intensity of the corresponding NMR line. This method gives rise to an enhancement of this line: upon complete conversion of the population difference into the coherence, the intensity of the forbidden transition increases by a factor of about 6.4 for ${ }^{13} \mathrm{C}_{2}$-I, as shown previously. ${ }^{32}$ At the same time, the 


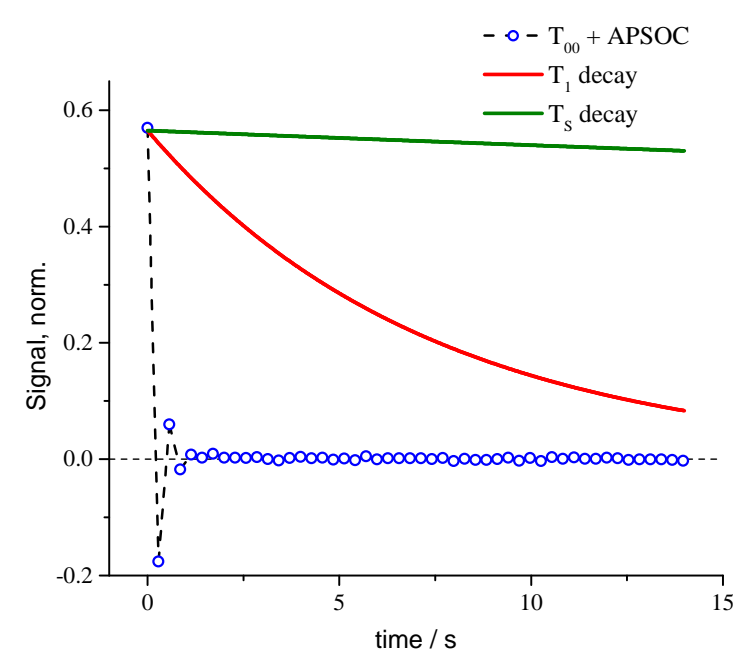

FIG. 6. Comparison of the experimental singlet order decay curves obtained using SOD (blue circles) with monoexponential curves, decaying with characteristic times $T_{1}=7.14 \mathrm{~s}$ (red line) and $T_{S}=220 \mathrm{~s}$ (green line).

populations of the $|S\rangle$ and $\left|T_{+}\right\rangle$states become different from their equilibrium values and singlet order is generated. For this reason, repetition of experiments of this kind require that both magnetization and singlet order relax back to their equilibrium values before the next experiment.

The results of the nutation experiments are shown in Figure 7. In this case, the nutation pattern is a sine function with its period determined by the RF-field strength; upon complete conversion of the population difference into the coherence a significant enhancement of the NMR line intensity is found, which is about 6 . The simplest way to achieve equilibrium values of both magnetization and singlet order is to introduce a relaxation delay of about $3 \cdot T_{S}$ to $5 \cdot T_{S}$. However, this straightforward method is impractical when $T_{S}$ is long: in the present case, the delay of $5 \cdot T_{S}$ would correspond to approximately $1200 \mathrm{~s}$ rendering the repetition time very long. At the same time, complete relaxation of singlet order is a pre-requisite for accurate measurement of the nutation curve: when the delay between subsequent experiments is $5 \cdot T_{1}$ (which is only $35 \mathrm{~s}$, i.e., much less than $T_{S}$ ) the nutation patterns are strongly distorted and the maximal NMR line enhancement drops by approximately a factor of 2 . In this situation, the proposed SOD method gives a remedy: one can obtain exactly the same nutation curve as for a very long delay but using a much shorter 
repetition time. In the example shown in Figure 7 the duration of the SOD block is only $7 \mathrm{~s}$, and it is followed by a delay of $5 \cdot T_{1}$. Hence, a reduction of the total time required to run the experiment is given by approximately a factor of $T_{S} / T_{1} \approx 30$. This example clearly shows that the SOD methods proposed here can significantly speed up experiments with long-lived singlet order.

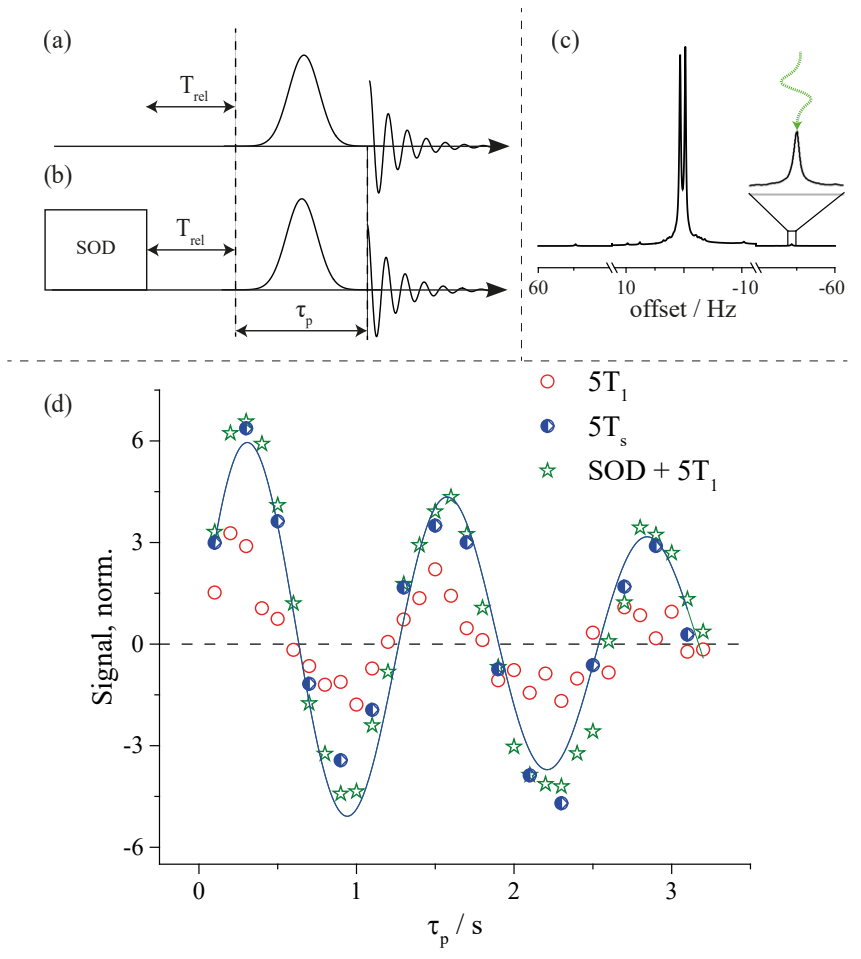

FIG. 7. Nutation patterns for excitation of a single "forbidden" transition obtained using a selective Gaussian pulse. (a) Pulse sequence with a relaxation period of $5 \cdot T_{1}$ between subsequent measurements. (b) Pulse sequence using the SOD block prior to the relaxation delay. (c) NMR spectrum with the forbidden transition indicated. (d) Nutation patterns obtained by using the pulse sequences (a) (red circles) and (b) (green stars, blue half-filled circles). The SOD block duration is $7 \mathrm{~s}$ (corresponding to 18 repetitions of SOD); the frequency of the pulse was set equal to the frequency of the high-field forbidden transition; the same phasing was used to obtain all NMR spectra. For each point, 4 scans were performed. All spectra were normalized by the line intensity in the thermal NMR spectrum; the resulting spectrum comes from 128 acquisitions using a $12 \mathrm{~Hz}$ Gaussian pulse of $0.3 \mathrm{~s}$ duration. The solid line shows the simulation by a decaying sine function. 


\section{Singlet order decay measurements}

Perhaps, the most obvious application of the SOD method is given by $T_{S}$ measurements. Such experiments are commonly done in the following way: one generates singlet order using an M2S conversion element, let the population imbalance relax during a time period $\tau_{e v}$, eliminate any residual spin order by using the $T_{00}$ filter, convert the singlet order back to magnetization and measure the NMR signal. By varying the evolution time we can get a monoexponetial decay curve: by fitting the relaxation trace we determine the conversion efficiency and the $T_{S}$ decay time. One should note, however, that application of the outlined pulse sequence can generate non-thermal singlet order, which persists if the delay between subsequent experiments is not sufficiently long, and may affect subsequent measurements. To avoid such experimental artefacts and reduce the time between the experiments, we propose to use the SOD sequence followed by a much shorter delay of $5 \cdot T_{1}$. The result of a $T_{S}$ measurement using the SOD method is shown in Figure 8.

It is important to note that the experimental curves obtained using either a long waiting time of $5 \cdot T_{S}$ or the SOD block between subsequent experiments are exactly the same, i.e., they are monoexponentially decaying curves. When the waiting time is reduced to $5 \cdot T_{1}$ and the SOD block is not used, there is a small rise of the singlet order at short $\tau_{e v}$ times, i.e., the shape of the relaxation trace is distorted. Here we refrain from a detailed analysis of this effect and only provide a brief intuitive explanation of the experimental finding. To analyze the observed effect, we assume that $\tau_{e v}=0$, i.e., that the $T_{00}$ filter is applied immediately after the APSOC pulse. At thermal equilibrium the state populations are equal to

$$
P_{T_{+}}=\frac{1}{4}(1+\Delta) ; \quad P_{T_{-}}=\frac{1}{4}(1-\Delta) ; \quad P_{T_{0}}=P_{S}=\frac{1}{4}
$$

where $\Delta=\frac{\hbar \gamma B_{0}}{k T}$ is the Boltzmann factor, $\gamma$ is the nuclear gyromagnetic ratio. After the $T_{+} \rightarrow$ $S$ conversion step followed by application of the $T_{00}$ filter the average triplet population, $\left\langle P_{T}\right\rangle$, is smaller than that at thermal equilibrium, being equal to $\left\langle P_{T}\right\rangle=\frac{1-P_{S}}{3}=\frac{1}{4}\left(1-\frac{\Delta}{3}\right)$. After the $S \rightarrow T_{+}$conversion step, the situation is opposite: $P_{S}=\frac{1}{4}\left(1-\frac{\Delta}{3}\right)$, i.e., the singlet state is underpopulated and $\left\langle P_{T}\right\rangle=\frac{1-P_{S}}{3}=\frac{1}{4}\left(1+\frac{\Delta}{9}\right)$, i.e., the mean triplet population is greater than that at equilibrium conditions. During the delay of $5 \cdot T_{1}$ triplet relaxation takes place. If we neglect singlet state relaxation and assume complete relaxation in the triplet manifold, the population of the $T_{+}$state can be written as $P_{T_{+}}=\left\langle P_{T}\right\rangle(1+\Delta)=\frac{1}{4}\left(1+\frac{11 \Delta}{9}\right)$, which is greater than the value at thermal equilibrium (7). Hence, singlet order is increased 


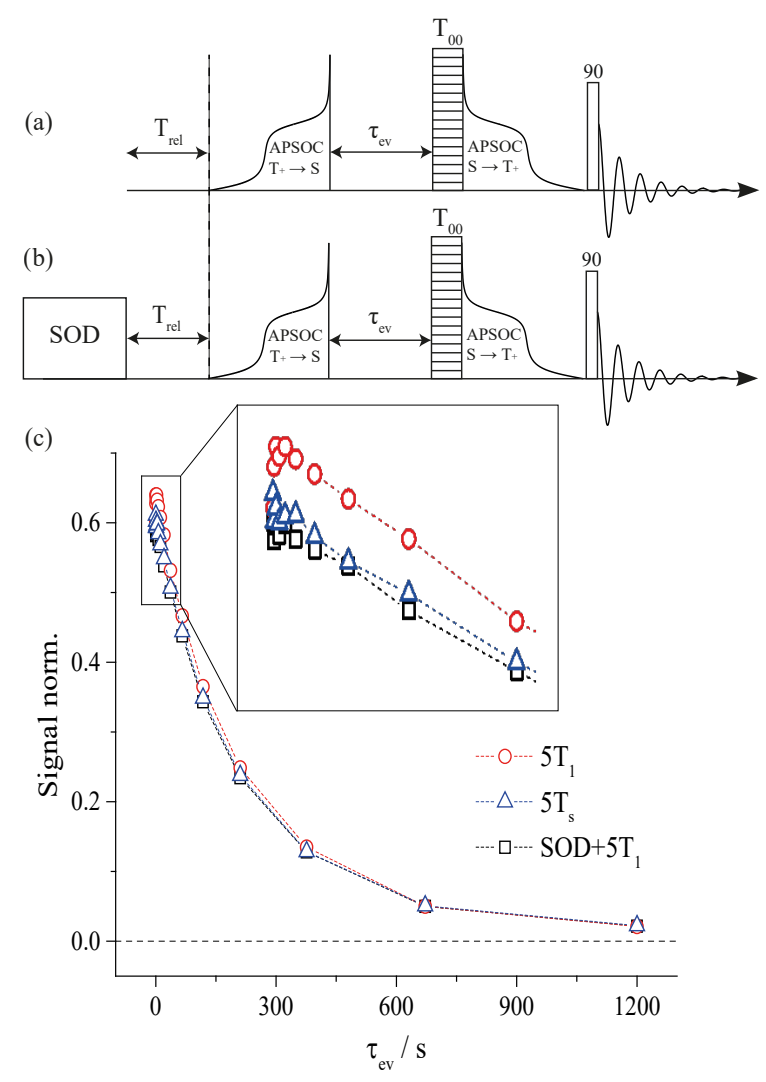

FIG. 8. Singlet order decay obtained using the APSOC sequence. (a) Pulse sequence with a relaxation period of $5 \cdot T_{1}$ or $5 \cdot T_{S}$ between subsequent measurements. (b) Pulse sequence using the SOD block and a relaxation delay of $5 \cdot T_{1}$; here the SOD block of a duration of $7 \mathrm{~s}$ consists of 18 cycles with a APSOC pulse followed by application of the $T_{00}$ filter. (c) Singlet order decay time trace obtained by using the pulse sequences (a) (red circles and blue triangles) and (b) (black squires). The signal intensity is normalized to that of the thermal NMR spectrum. The parameters of APSOC pulse are the same as in Fig. 3.

after the subsequent $S \rightarrow T_{+}$conversion step, which is in agreement with the experimental observation.

\section{SUMMARY}

In this work, we propose efficient schemes for singlet order destruction in pairs of nearly equivalent spins. These schemes utilize singlet-triplet conversion and an element (a field gradient or $T_{00}$-filter), which removes residual spin order in the triplet manifold. We are able 
to demonstrate that the times required to wipe out the singlet order are much shorter than the $T_{S}$ relaxation time and are even shorter than the $T_{1}$ relaxation time. Consequently, such schemes can be used to reduce dramatically the total time of experiments with long-lived singlet order by minimizing the relaxation delay between subsequent measurements. Hence, we suggest to use the SOD techniques as blocks in pulse sequences used in singlet-state NMR experiments dealing with pairs of nearly equivalent spins. We can clearly demonstrate that without SOD such experiments take unreasonably long time or lead to artefacts or lack of reproducibility. Strongly coupled spin pairs can be encountered in molecules specially designed for experiments utilizing remarkable properties of singlet states or in experiments performed at low fields, where the differences in NMR frequencies are reduced and become smaller than J-coupling.

\section{SUPPLEMENTARY MATERIAL}

See Supplementary Material for discussion of SLIC-based singlet order destruction and implementation of the triplet equalization element in the SOD sequence.

\section{ACKNOWLEDGMENTS}

This work has been supported by the Russian Foundation for Basic Research (grant No. 19-32-80004), EPSRC-UK (grant reference EP/P009980/1) and European Research Council (786707-FunMagResBeacons). We acknowledge the Russian Ministry of Science and Higher Education (project AAAA-A16-116121510087-5) for providing access to NMR facilities at ITC SB RAS.

\section{REFERENCES}

${ }^{1}$ M. H. Levitt, "Singlet Nuclear Magnetic Resonance," in Annu. Rev. Phys. Chem., Annual Review of Physical Chemistry, Vol. 63, edited by M. A. Johnson and T. J. Martinez (Annual Reviews, Palo Alto, 2012) pp. 89-105.

${ }^{2}$ M. Carravetta, O. G. Johannessen, and M. H. Levitt, "Beyond the $T_{1}$ Limit: Singlet Nuclear Spin States in Low Magnetic Fields," Physical Review Letters 92, 153003 (2004).

${ }^{3}$ M. Carravetta and M. H. Levitt, "Long-Lived Nuclear Spin States in High-Field Solution NMR," Journal of the American Chemical Society 126, 6228-6229 (2004). 
${ }^{4}$ G. Stevanato, J. T. Hill-Cousins, P. Hakansson, S. S. Roy, L. J. Brown, R. C. D. Brown, G. Pileio, and M. H. Levitt, "A Nuclear Singlet Lifetime of More than One Hour in Room-Temperature Solution," Angewandte Chemie, International Edition 54, 3740-3743 (2015).

${ }^{5}$ G. Pileio, M. Carravetta, E. Hughes, and M. H. Levitt, "The long-lived nuclear singlet state of N-15-nitrous oxide in solution," Journal of the American Chemical Society 130, $12582-+(2008)$.

${ }^{6}$ A. S. Kiryutin, S. E. Korchak, K. L. Ivanov, A. V. Yurkovskaya, and H.-M. Vieth, "Creating Long-Lived Spin States at Variable Magnetic Field by Means of Photochemically Induced Dynamic Nuclear Polarization," Journal of Physical Chemistry Letters 3, 1814 1819 (2012).

${ }^{7}$ R. Sarkar, P. R. Vasos, and G. Bodenhausen, "Singlet-state exchange NMR spectroscopy for the study of very slow dynamic processes," Journal of the American Chemical Society 129, 328-334 (2007).

${ }^{8}$ S. Cavadini, J. Dittmer, S. Antonijevic, and G. Bodenhausen, "Slow Diffusion by Singlet State NMR Spectroscopy," Journal of the American Chemical Society 127, 15744-15748 (2005).

${ }^{9}$ G. Pileio, J. N. Dumez, I. A. Pop, J. T. Hill-Cousins, and R. C. D. Brown, "Realspace imaging of macroscopic diffusion and slow flow by singlet tagging MRI," Journal of Magnetic Resonance 252, 130-134 (2015).

${ }^{10}$ R. Sarkar, P. Ahuja, P. R. Vasos, and G. Bodenhausen, "Measurement of Slow Diffusion Coefficients of Molecules with Arbitrary Scalar Couplings via Long-Lived Spin States," Chemphyschem 9, 2414-2419 (2008).

${ }^{11}$ P. Ahuja, R. Sarkar, P. R. Vasos, and G. Bodenhausen, "Diffusion Coefficients of Biomolecules Using Long-Lived Spin States," Journal of the American Chemical Society 131, 7498-7499 (2009).

${ }^{12}$ R. Buratto, D. Mammoli, E. Chiarparin, G. Williams, and G. Bodenhausen, "Exploring Weak Ligand-Protein Interactions by Long-Lived NMR States: Improved Contrast in Fragment-Based Drug Screening," Angewandte Chemie, International Edition 53, 1137611380 (2014).

${ }^{13}$ N. Salvi, R. Buratto, A. Bornet, S. Ulzega, I. R. Rebollo, A. Angelini, C. Heinis, and G. Bodenhausen, "Boosting the Sensitivity of Ligand-Protein Screening by NMR of Long- 
Lived States," Journal of the American Chemical Society 134, 11076-11079 (2012).

${ }^{14}$ Y. N. Zhang, P. C. Soon, A. Jerschow, and J. W. Canary, "Long- Lived 1h Nuclear Spin Singlet in Dimethyl Maleate Revealed by Addition of Thiols," Angewandte Chemie, International Edition 53, 3396-3399 (2014).

${ }^{15}$ P. Ahuja, R. Sarkar, S. Jannin, P. R. Vasos, and G. Bodenhausen, "Proton hyperpolarisation preserved in long-lived states." Chemical Communications 46, 8192-4 (2010).

${ }^{16}$ P. R. Vasos, A. Comment, R. Sarkar, P. Ahuja, S. Jannin, J. P. Ansermet, J. A. Konter, P. Hautle, B. Van Den Brandt, and G. Bodenhausen, "Long-lived states to sustain hyperpolarized magnetization," Proceedings of the National Academy of Sciences of the United States of America 106, 18469-18473 (2009).

${ }^{17}$ G. Pileio, S. Bowen, C. Laustsen, M. C. D. Tayler, J. T. Hill-Cousins, L. J. Brown, R. C. D. Brown, J. H. Ardenkjaer-Larsen, and M. H. Levitt, "Recycling and Imaging of Nuclear Singlet Hyperpolarization,” Journal of the American Chemical Society 135, 5084-5088 (2013).

${ }^{18}$ B. Meier, J. N. Dumez, G. Stevanato, J. T. Hill-Cousins, S. S. Roy, P. Hakansson, S. Mamone, R. C. D. Brown, G. Pileio, and M. H. Levitt, "Long-Lived Nuclear Spin States in Methyl Groups and Quantum-Rotor-Induced Polarization," Journal of the American Chemical Society 135, 18746-18749 (2013).

${ }^{19}$ M. B. Franzoni, D. Graafen, L. Buljubasich, L. M. Schreiber, H. W. Spiess, and K. Münnemann, "Hyperpolarized H-1 long lived states originating from parahydrogen accessed by rf irradiation," Physical Chemistry Chemical Physics 15, 17233-17239 (2013).

${ }^{20}$ M. B. Franzoni, L. Buljubasich, H. W. Spiess, and K. Münnemann, "Long-Lived 1h Singlet Spin States Originating from Para-Hydrogen in Cs-Symmetric Molecules Stored for Minutes in High Magnetic Fields," Journal of the American Chemical Society 134, 10393-10396 (2012).

${ }^{21}$ K. L. Ivanov, T. Kress, M. Baudin, D. Guarin, D. Abergel, G. Bodenhausen, and D. Kurzbach, "Relaxation of long-lived modes in NMR of deuterated methyl groups," Journal of Chemical Physics 149, 054202 (2018).

${ }^{22}$ T. Theis, G. X. Ortiz, A. W. J. Logan, K. E. Claytor, Y. Feng, W. P. Huhn, V. Blum, S. J. Malcolmson, E. Y. Chekmenev, Q. Wang, and W. S. Warren, "Direct and cost-efficient hyperpolarization of long-lived nuclear spin states on universal 15n2-diazirine molecular tags," Science Advances 2, e1501438 (2016). 
${ }^{23}$ Y. Feng, T. Theis, T. L. Wu, K. Claytor, and W. S. Warren, "Long-lived polarization protected by symmetry," Journal of Chemical Physics 141, 134307 (2014).

${ }^{24}$ S. J. Elliott, C. Bengs, L. J. Brown, J. T. Hill-Cousins, D. J. O'Leary, G. Pileio, and M. H. Levitt, "Nuclear singlet relaxation by scalar relaxation of the second kind in the slow-fluctuation regime," The Journal of Chemical Physics 150, 064315 (2019).

${ }^{25}$ Y. Feng, R. M. Davis, and W. S. Warren, "Accessing long-lived nuclear singlet states between chemically equivalent spins without breaking symmetry," Nature Physics 8, 831837 (2012).

${ }^{26}$ Y. Feng, T. Theis, X. Liang, Q. Wang, P. Zhou, and W. S. Warren, "Storage of Hydrogen Spin Polarization in Long-Lived 13c2 Singlet Order and Implications for Hyperpolarized Magnetic Resonance Imaging," Journal of the American Chemical Society 135, 9632-9635 (2013).

${ }^{27}$ K. F. Sheberstov, H.-M. Vieth, H. Zimmermann, K. L. Ivanov, A. S. Kiryutin, and A. V. Yurkovskaya, "cis Versus trans-Azobenzene: Precise Determination of NMR Parameters and Analysis of Long-Lived States of 15n Spin Pairs," Applied Magnetic Resonance 49, 293-307 (2018).

${ }^{28}$ M. C. D. Tayler and M. H. Levitt, "Singlet nuclear magnetic resonance of nearly-equivalent spins," Physical Chemistry Chemical Physics 13, 5556-5560 (2011).

${ }^{29}$ S. J. DeVience, R. L. Walsworth, and M. S. Rosen, "Preparation of Nuclear Spin Singlet States Using Spin-Lock Induced Crossing," Physical Review Letters 111, 173002 (2013).

${ }^{30}$ A. N. Pravdivtsev, A. S. Kiryutin, A. V. Yurkovskaya, H.-M. Vieth, and K. L. Ivanov, "Robust conversion of singlet spin order in coupled spin-1/2 pairs by adiabatically ramped RF-fields," Journal of Magnetic Resonance 273, 56-64 (2016).

${ }^{31}$ A. Kiryutin, H. Zimmermann, A. Yurkovskaya, H.-M. Vieth, and K. Ivanov, "Long-lived spin states as a source of contrast in magnetic resonance spectroscopy and imaging," Journal of Magnetic Resonance 261, 64-72 (2015).

${ }^{32}$ K. F. Sheberstov, A. S. Kiryutin, C. Bengs, J. Hill-Cousins, L. J. Brown, R. Brown, G. Pileio, M. H. Levitt, A. V. Yurkovskaya, and K. L. Ivanov, "Excitation of singlet-triplet coherences in pairs of nearly-equivalent spins," Physical Chemistry Chemical Physics 21, 6087-6100 (2019).

${ }^{33}$ B. A. Rodin, K. F. Sheberstov, A. S. Kiryutin, J. T. Hill-Cousins, L. J. Brown, R. C. D. Brown, B. Jamain, H. Zimmermann, R. Z. Sagdeev, A. V. Yurkovskaya, and K. L. Ivanov, 
"Constant-adiabaticity RF-pulses for generating long-lived singlet spin states in NMR," Journal of Chemical Physics 150, 064201 (2019).

${ }^{34}$ M. C. D. Tayler and M. H. Levitt, "Accessing Long-Lived Nuclear Spin Order by IsotopeInduced Symmetry Breaking," Journal of the American Chemical Society 135, 2120-2123 (2013).

${ }^{35}$ S. Mamone and S. Glöggler, "Nuclear spin singlet states as magnetic on/off probes in self-assembling systems," Physical Chemistry Chemical Physics 20, 22463-22467 (2018).

${ }^{36}$ A. S. Kiryutin, K. L. Ivanov, A. V. Yurkovskaya, H.-M. Vieth, and N. N. Lukzen, "Manipulating spin hyper-polarization by means of adiabatic switching of a spin-locking RF-field," Physical Chemistry Chemical Physics 15, 14248-14255 (2013).

${ }^{37}$ B. A. Rodin, A. S. Kiryutin, A. V. Yurkovskaya, K. L. Ivanov, S. Yamamoto, K. Sato, and T. Takui, "Using optimal control methods with constraints to generate singlet states in NMR," Journal of Magnetic Resonance 291, 14-22 (2018). 
(a)

(b)

$\mathrm{SO}=3$

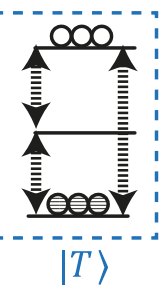

Triplet Equalization
$\mathrm{T} \leftrightarrow \mathrm{S}$
Triplet

Equalization

$$
\mathrm{SO}=-1
$$

en

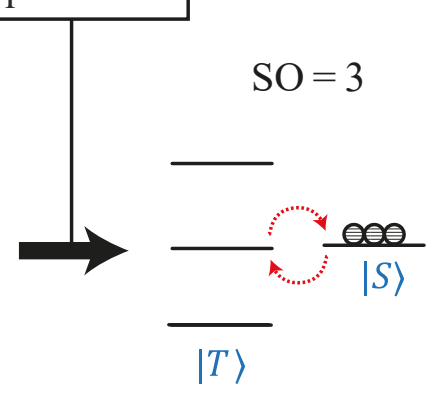

$|S\rangle$

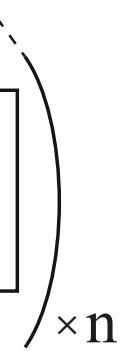

$|S\rangle$

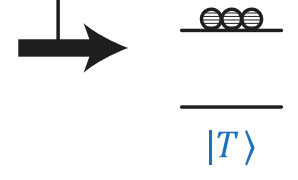

\section{$\mathrm{T} \leftrightarrow \mathrm{S}$

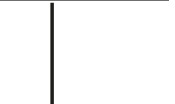

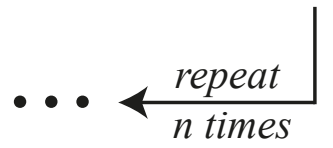




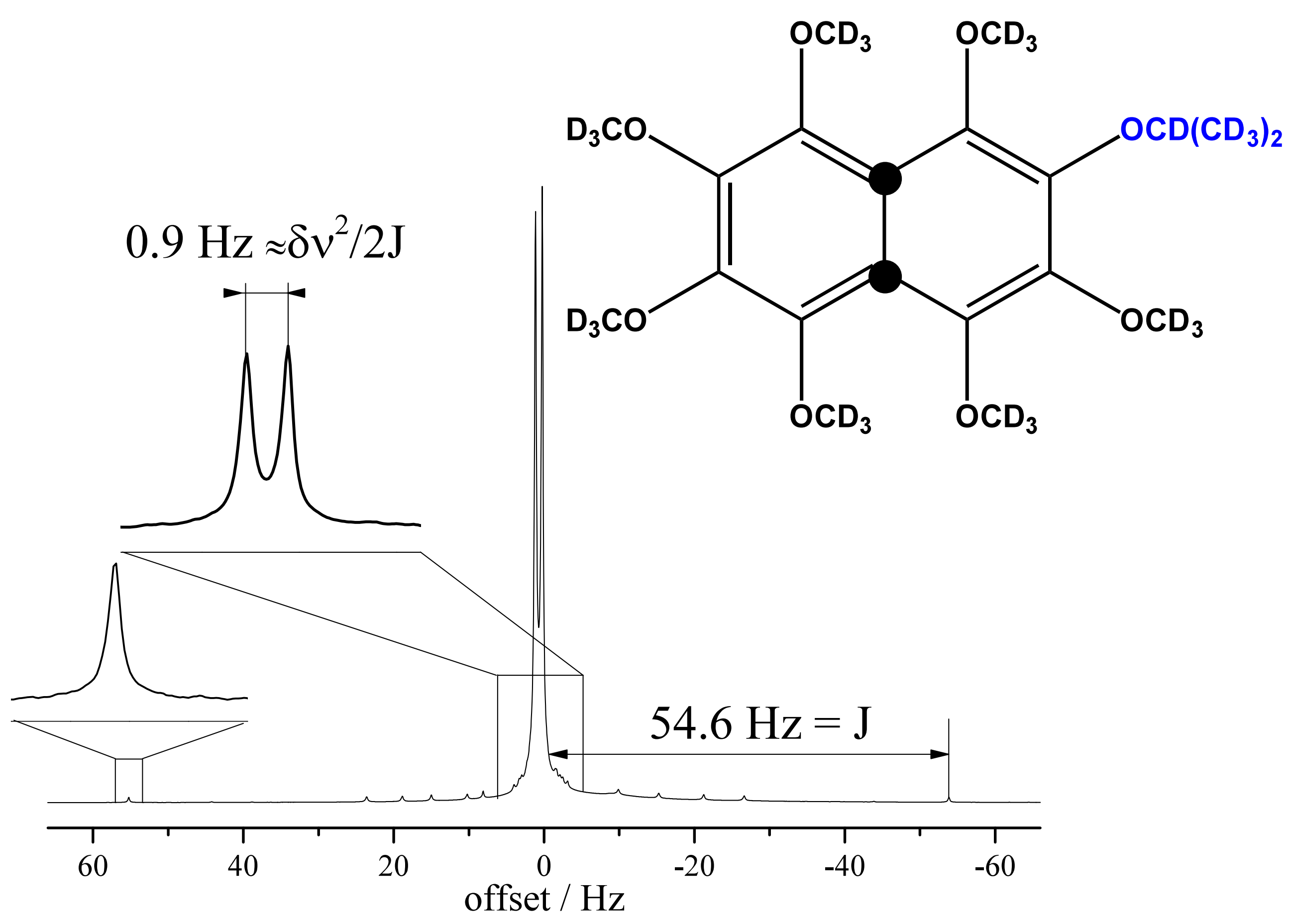




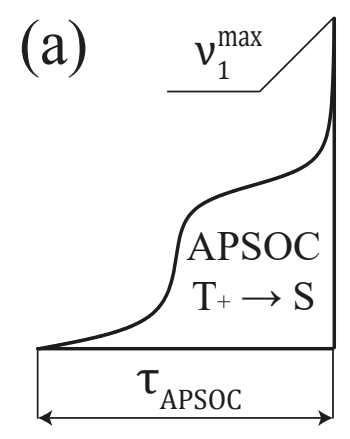

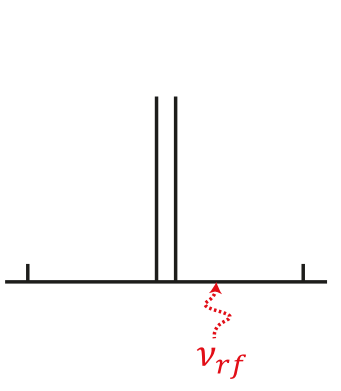

$90_{0} 180_{90} 90_{0}$
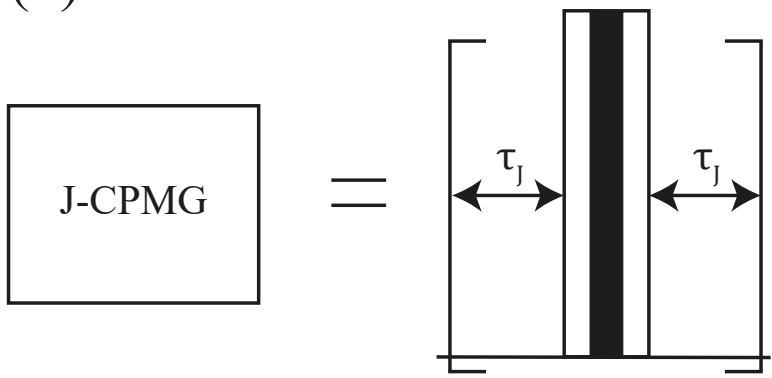

$\times \mathbf{n}_{\mathrm{J}}$

(c)
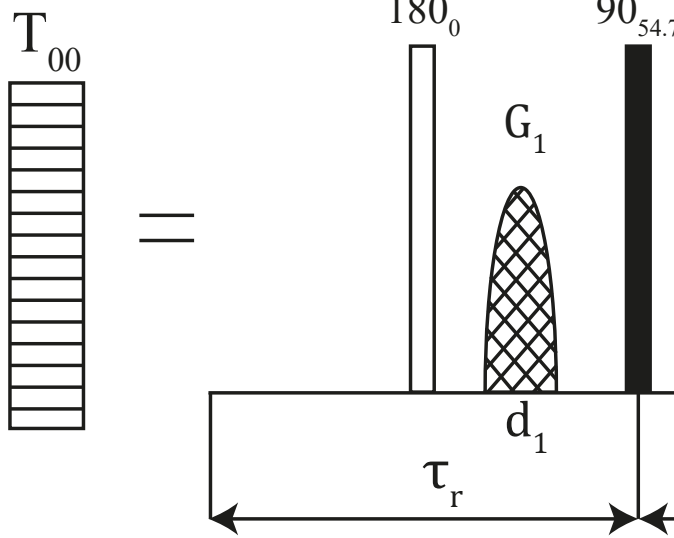

$\overline{180} \overline{0}_{0}$
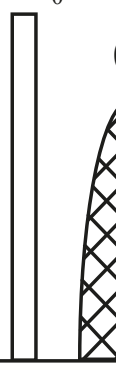

(3)

$\tau$
$9 \overline{0}_{54.735}-\overline{90}--\overline{180}-\overline{0}$

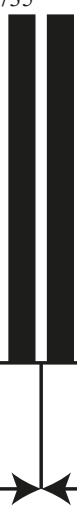

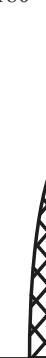

$\mathrm{d}_{3}$

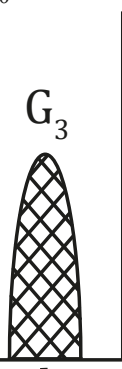

$\tau_{r}$ 



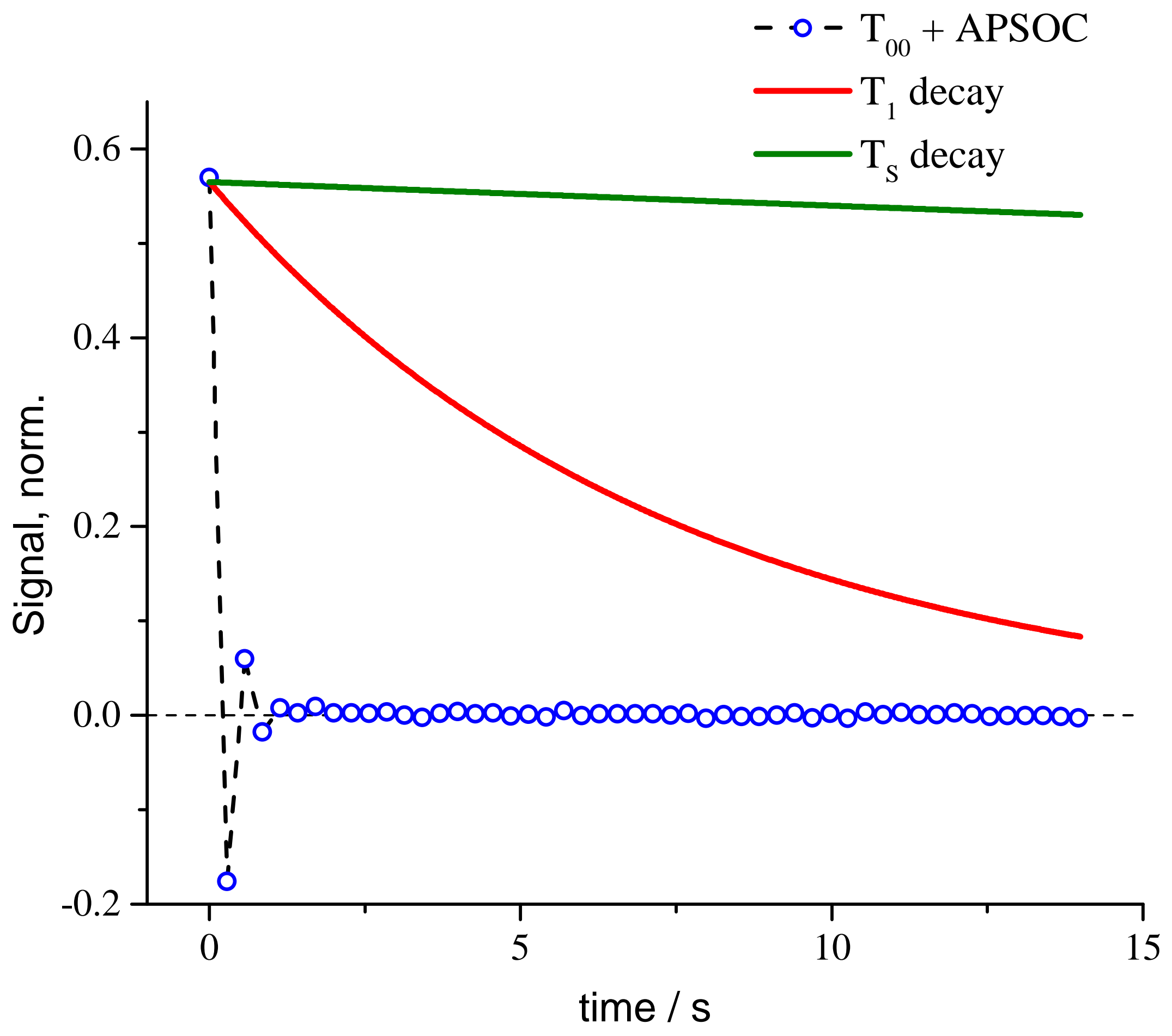



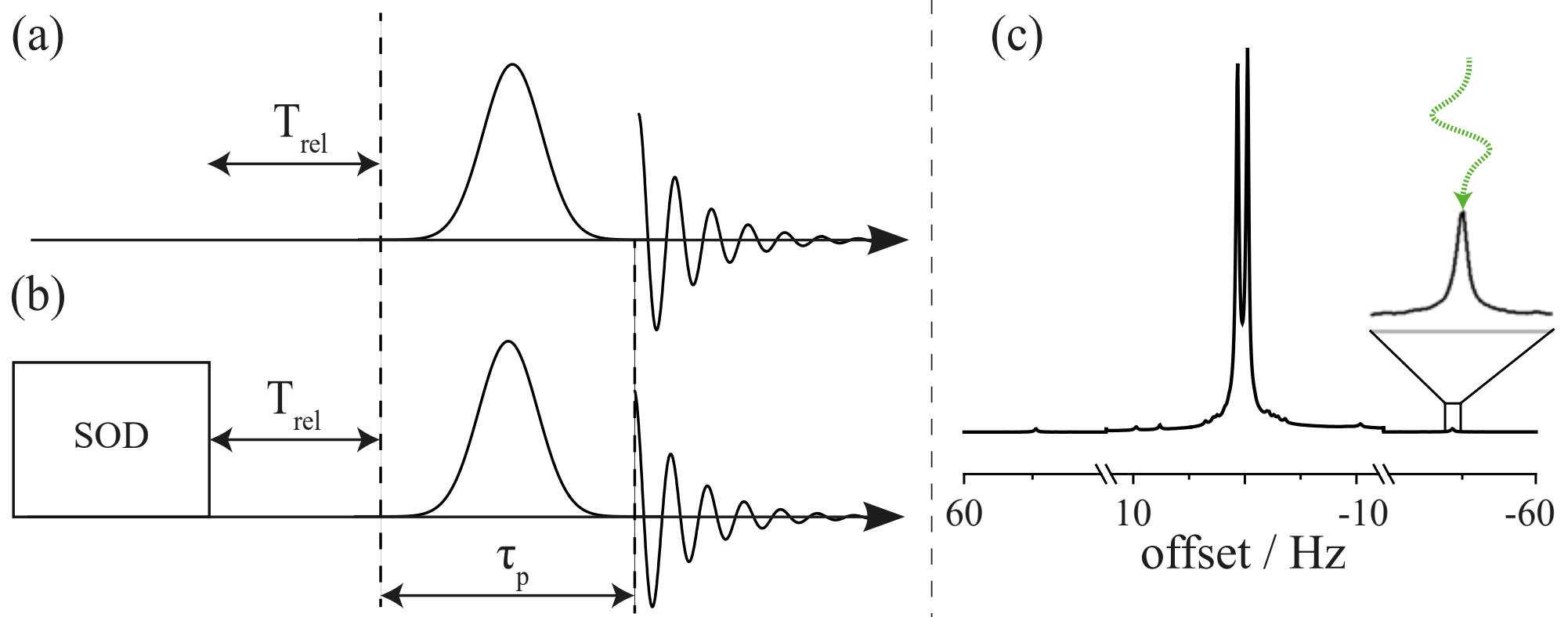

(d)

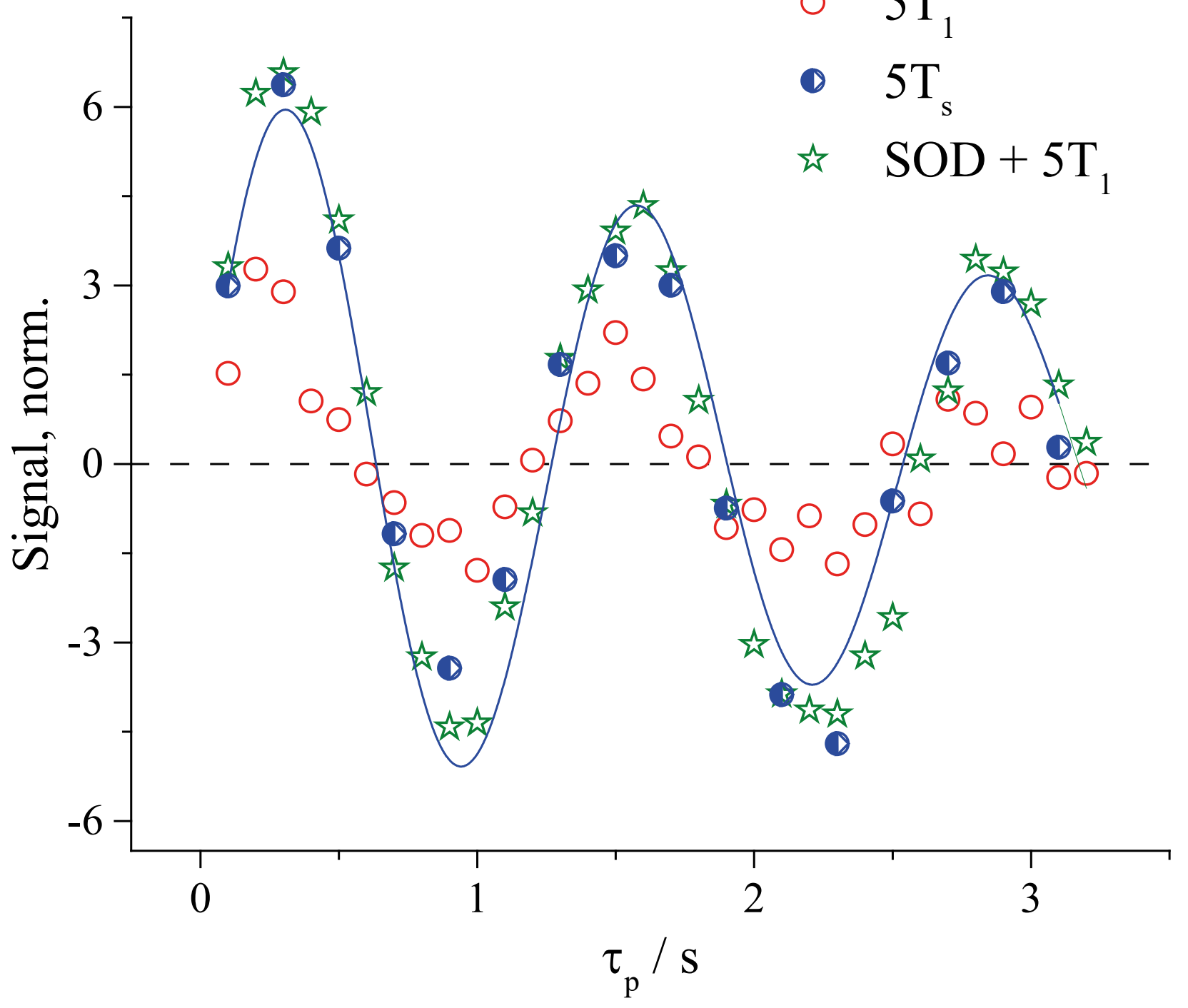




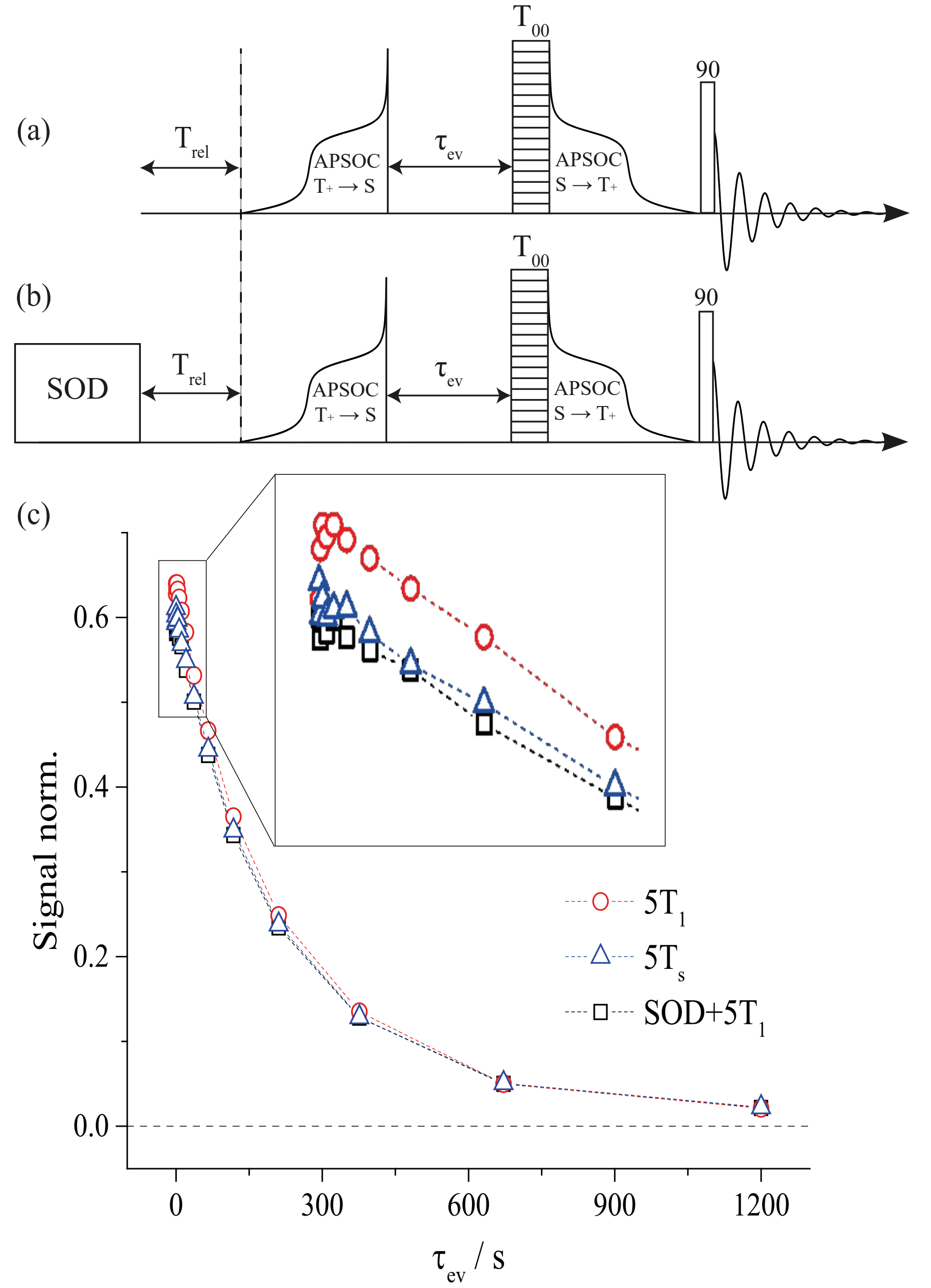

